\title{
Larvicidal activity of essential oil from Vitex negundo and Vitex trifolia on dengue vector mosquito Aedes aegypti
}

\author{
Trilokesh Chandrasekaran ${ }^{[1]}$, Aishwarya Thyagarajan ${ }^{[1]}$, Prabhakaran Ganapathi Santhakumari ${ }^{[1]}$, \\ Agiesh Kumar Balakrishna Pillai ${ }^{[2]}$ and Uma Maheswari Krishnan ${ }^{[1],[3]}$
}

\author{
[1]. School of Chemical and Biotechnology (SCBT), SASTRA Deemed to be University, India. \\ [2]. Central Inter Disciplinary Research Facility (CIDRF), Sri Balaji Vidyapeeth, Deemed to be University, Puducherry, India. \\ [3]. Centre for Nanotechnology and Advanced Biomaterials, SASTRA Deemed to be University (CeNTAB), India.
}

\begin{abstract}
Introduction: The ability of Vitex trifolia and Vitex negundo essential oils to kill Aedes aegypti and Culex quinquefasciatus larvae was evaluated. Methods: The larvae were treated with their respective essential oils at 50-125 ppm concentration. Results: $\mathrm{LC}_{50}$ and $\mathrm{LC}_{90}$ for $V$. trifolia against Ae. aegypti and C. quinquefasciatus, and those for $V$. negundo against Ae. aegypti were 57.7 \pm 0.4 , $77.9 \pm 0.9 \mathrm{ppm}$ and $55.17 \pm 3.14,78.28 \pm 2.23 \mathrm{ppm}$, and $50.86 \pm 0.9,73.12 \pm 1.3 \mathrm{ppm}$, respectively. Eucalyptol and caryophyllene were the major components in Vitex trifolia and Vitex negundo essential oil, respectively. Conclusions: This study revealed potential larvicidal properties of essential oil from V. trifolia.
\end{abstract}

Keywords: Aedes aegypti. Culex quinquefasciatus. Vitex trifolia. Vitex negundo. Essential oil.

Of all arthropods, mosquitoes are the most essential for medical research, because they are vectors for diseases such as malaria, yellow fever, dengue fever, chikungunya fever, filaria, encephalitis, and West Nile virus infection. Mosquito-borne diseases are prevalent in almost all tropical and subtropical countries, as well as many other parts of the world ${ }^{1}$. Although there are several disease vector mosquitoes, only three of them are considered dangerous: Aedes aegypti, which transmits the dengue virus, Culex quinquefasciatus, which harbors and transmits the West Nile Virus, and finally Anopheles gambiae, which transmits deadly malarial parasites ${ }^{1}$. Among the genus Aedes, the troublesome species include Ae. aegypti and Ae. Albopictus, which transmit diseases such as dengue, zika, and chikungunya. In the genus Culex, the dominant diseasecausing species are C. quinquefasciatus, C. pipiens, and C. tritaeniorynchus, which transmit diseases such as filaria, avian malaria, Japanese encephalitis, and West Nile virus. Since no vaccines or effective drugs have been developed, to combat dengue or other mosquito-borne viral diseases to date,

Corresponding authors: Uma Maheswari Krishnan, Agiesh Kumar Balakrishna Pillai. e-mail: umakrishnan@sastra.edu, agiesh.b@gmail.com Orcid: 0000-0001-6508-4485, 0000-0002-6543-2977

Received 24 October 2018

Accepted 28 January 2019 mosquito control remains the primary strategy for controlling mosquito-borne diseases. Control measures rely mainly on chemical insecticides that target mosquitoes. Although synthetic repellents and larvicides are present in the market, they fail to produce significant protection against mosquitoes; synthetic repellents also have side effects. Overuse of synthetic insecticides results in increased resistance among mosquito species $^{1}$. One of the most effective alternative approaches to finding a safer, sustainable, and straightforward insecticide for mosquito control, is to explore the floral biodiversity.

Furthermore, the chance of mosquitoes developing resistance towards plant metabolites is very little. Plant-derived insecticides comprise of a cocktail of compounds that may have a synergistic action on killing mosquitoes, unlike conventional pesticides that are based on a single active ingredient ${ }^{2}$. In this context, the present study reports the mosquito larvicidal properties of the essential oils extracted from the leaves of Vitex trifolia (V. trifolia) and Vitex negundo ( $V$. negundo), which are shrubs belonging to the family Lamiaceae.

Plant materials used in the study were collected from the herbal garden of SASTRA Deemed University, Thanjavur, Tamil Nadu 613401, India. The collected plants were identified and authenticated with the help of Flora of Tamil Nadu Carnatic Herbarium available in the Rapinet Herbarium, St. Joseph's College ${ }^{3}$, Trichy; the specimen numbers are RHT 7317 and RHT 
493 for $V$. trifolia, and $V$. negundo, respectively. The leaves were weighed and collected, and essential oils were extracted by steam distillation using a Clevenger apparatus. The oils were collected in glass vials after $4-5 \mathrm{~h}$ of distillation (Figure 1). A pinch of anhydrous sodium sulphate was added to remove any residual moisture in the oil and was then stored for one week at $-4{ }^{\circ} \mathrm{C}$ in airtight vials to avoid evaporation until further trials. A total of $273 \mathrm{~g}$ of leaves from both the plants yielded 5\% extracted oils. The essential oil was analyzed for its chemical composition using gas chromatography-mass spectrometry (GC-MS). The retention index (RI) of the gas chromatography peaks was compared using a homologous series of n-alkanes (C8-C20) for identifying components of the essential oil. The mass spectra obtained were compared with standard spectra and the NIST 2005 MS library.
The Aedes and Culex egg rafts were collected from different breeding grounds and area of stagnant water puddles in Thanjavur, Tamil Nadu, India. They were fed with a ratio of brewer's yeast to dog's biscuit of 1:3. After 2-3 days, the eggs hatched and the early stage larvae ( $3^{\text {rd }}$ instar) were taken for the study. Larvicidal activities of the essential oils were carried out as per the standard protocol of the World Health Organization ${ }^{4}$. A sufficient amount of target oil was stabilized, using $100 \%$ acetone, to produce a stock solution. The bioassay was carried out for 20 of the $3^{\text {rd }}$ instar larvae of Ae. aegypti and C. quinquefasciatus, which were taken in disposable cups $(250 \mathrm{~mL})$ containing $100 \mathrm{~mL}$ of tap water. From the prepared stock of the oil, different concentrations, ranging from 50-125 ppm, were added to the beakers. The larvicidal activity of $V$. trifolia on Ae. aegypti and C. quinquefasciatus, and that of

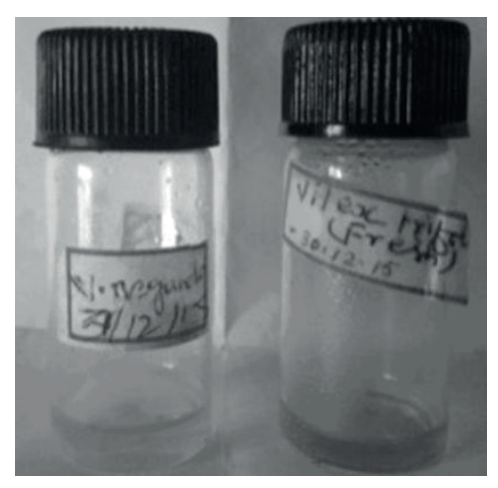

(A)

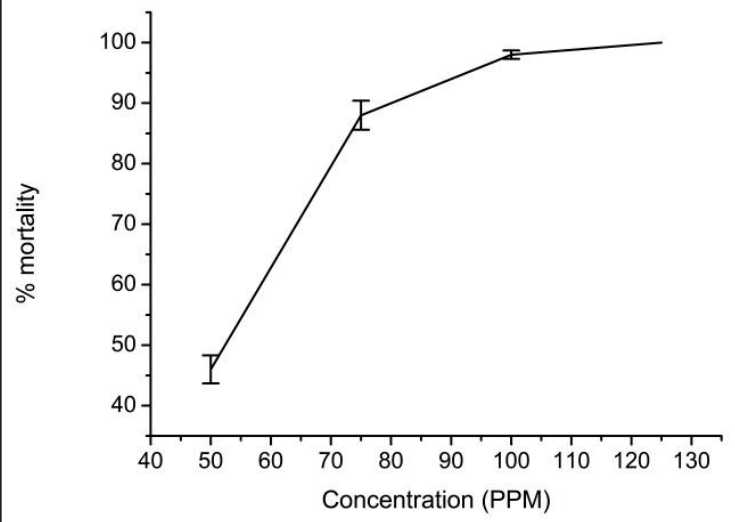

(C)

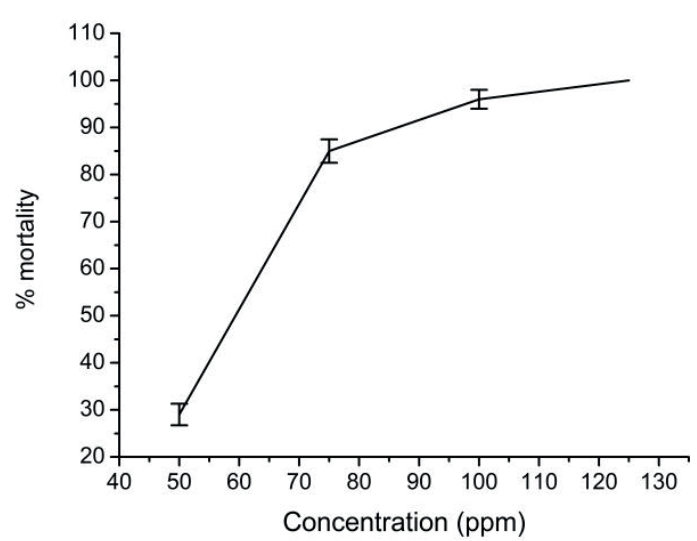

(B)

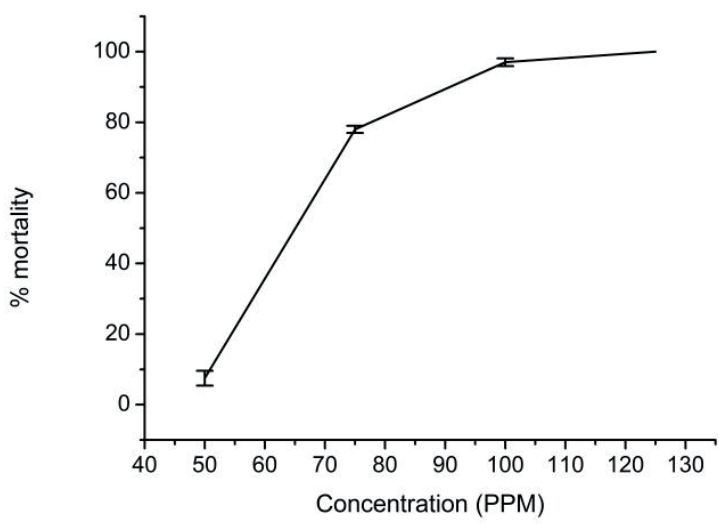

(D)

FIGURE 1: (A) Extracted essential oils of V. negundo and V. trifolia. (B) Effect of V. trifolia essential oil on $3^{\text {rd }}$ instar larvae of Aedes aegypti at concentrations of 50,75, 100, and 125 ppm. (C) Effect of $V$. negundo essential oil on $3^{\text {rd }}$ instar larvae of Aedes aegypti at concentrations of 50,75, 100, and 125 ppm. (D) Effect of V. trifolia essential oil on 3rd instar larvae of Culex quinquefasciatus at concentrations of 50, 75, 100 , and $125 \mathrm{ppm}$. 
$V$. negundo on Ae. aegypti were studied. For each experiment, a set of controls, using acetone and untreated larvae in tap water, were also maintained for comparison. The mortality of the larvae were observed over 24 hours post-treatment. Both dead and moribund larvae were classified as non-existent. Each trial was performed in duplicates. The mortality was observed and documented. The lethal concentrations, of $50 \%\left(\mathrm{LC}_{50}\right)$ and $90 \%\left(\mathrm{LC}_{90}\right)$, were calculated as the concentration $(\mathrm{ppm})$ required to kill $50 \%$ and $90 \%$ of the larvae population respectively.

The mortality data were analyzed statistically5. From the regression line, between logarithmic dose and mortality, $\mathrm{LC}_{50}$, and $\mathrm{LC}_{90}$ were determined at the $95 \%$ confidence interval. The regression line was plotted using Microsoft Excel.

GC-MS analysis of $V$. trifolia showed a total of 89 components (Figure 2A). The major component was identified as eucalyptol, a known insect repellent. Sabinen and caryophyllene were identified as the other main components. Regarding $V$. negundo, a total of 60 compounds were found (Figure 2B). The principal component of which was found to be caryophyllene, with a peak area of $27.984 \%$ and a retention time of $16.48 \mathrm{~min}$.

The bioassay results revealed a significant larvicidal activity $(\mathrm{P}<0.05)$ of the oils against Ae. aegypti and $C$. quinquefasciatus larvae (Table 1). Percentage mortalities after the addition of essential oil is shown in Figures 1A, 1B, and 1C. From the figures, it is clear that Culex quinquefasciatus is comparatively more susceptible than Ae. aegypti to the larvicidal activity of $V$. trifolia. The $\mathrm{LC}_{50}$ and $\mathrm{LC}_{90}$ values were calculated using probit analysis. The bioassay of the third instar stage of Aedes species showed an $\mathrm{LC}_{50}$ value of $57.7 \pm 0.4 \mathrm{ppm}$ and an $\mathrm{LC}_{90}$ value of $77.9 \pm 0.9 \mathrm{ppm}$ of oils from $V$. trifolia. The $\mathrm{LC}_{50}$ and $\mathrm{LC}_{90}$ values of $V$. trifolia on $C$. quinquefasciatus were $55.17 \pm 3.14$ and $78.28 \pm 2.23 \mathrm{ppm}$ respectively. The $\mathrm{LC}_{50}$ and $\mathrm{LC}_{90}$ values of $V$. negundo on Ae. aegypti was $50.86 \pm 0.9$ and $73.12 \pm 1.3 \mathrm{ppm}$ respectively. A concentration of $125 \mathrm{ppm} V$. trifolia oil was required to kill 20 Ae. aegypti and C. quinquefasciatus larvae.

Similarly, when the susceptibility of Ae. aegypti to $V$. negundo was evaluated, $125 \mathrm{ppm}$ concentration was required to kill the larvae. From these trials, it was found out that both $V$. negundo and $V$. trifolia had significant larvicidal activity against Ae. aegypti and C. quinquefasciatus.

Vitex species are commonly seen on the banks of water bodies like channels, rivers, and ponds. Many plants of this genus were reported to have high therapeutic values. Their dried leaves were said to be burnt to deter mosquitoes. Methanol extracts of the leaves from Vitex altissima, Vitex negundo, Vitex trifolia, and Vitex peduncularis were reported to possess potential mosquito larvicidal property against the larvae of C. quinquefasciatus. Among all the species of Vitex, V. trifolia possess the highest ability to control mosquito larvae ${ }^{7}$. Another study by the group analyzed the larvicidal activity of fatty acid methyl esters obtained from the leaf extract of Vitex altissima, Vitex negundo and Vitex trifolia. The study found the highest toxicity effect in the extract of $V$. trifolia against C. quinquefasciatus larvae $\left(\mathrm{LC}_{50}=9.26 \text { and } \mathrm{LC}_{90}=21.28 \mathrm{ppm}\right)^{8}$. In the same study, methyl-p-hydroxybenzoate was separated from the plant extract and studied for its toxic effects on the $4^{\text {th }}$ instar larvae of $C$. quinquefasciatus and Ae. aegypti. The compound exhibited a very high killing activity against mosquito larvae of both species at low concentration'. However, no studies have explored the mosquito larvicidal properties of the essential oil of the leaves of $V$. trifolia, except a study which reported the oviposition deterrent activity of the oil against adult mosquitoes ${ }^{10}$. Since mosquito larvae and pupa stages can be targeted effectively for mass control measures, unlike adult mosquitoes ${ }^{11}$, the present study assessed the efficacy of the essential oils of $V$. trifolia and $V$. negundo against the larvae of C. quinquefasciatus and Ae. aegypti.
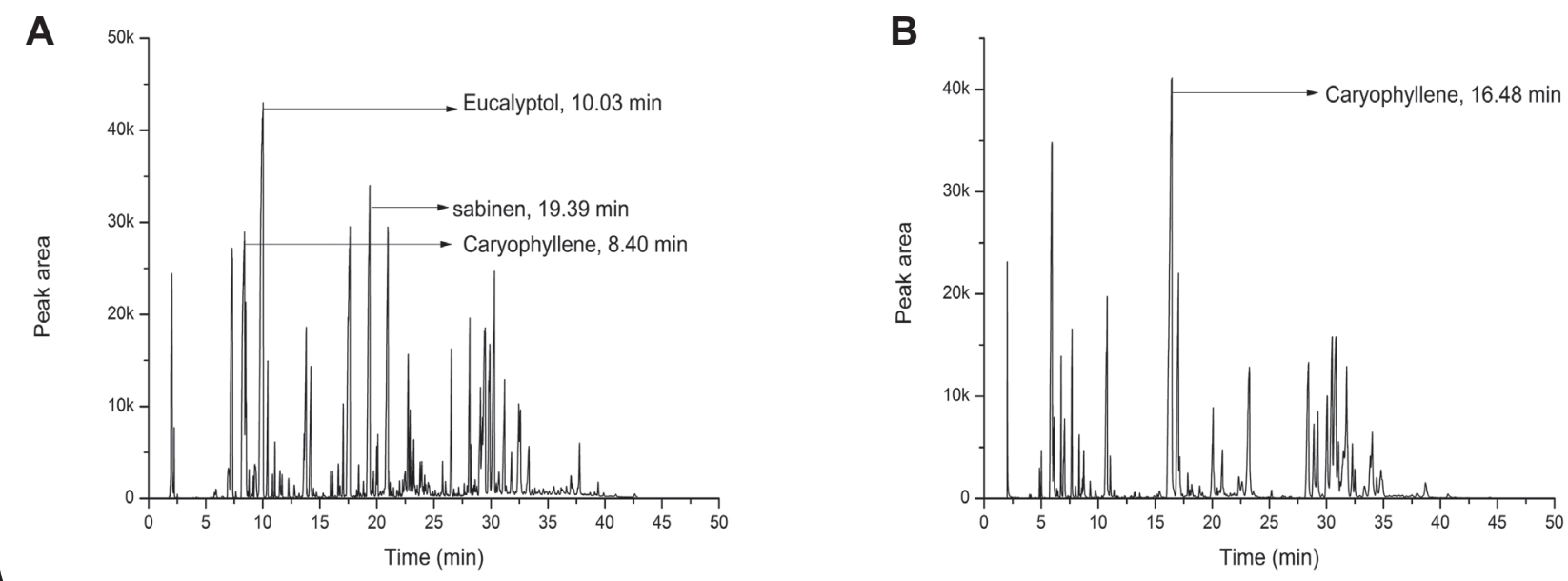

FIGURE 2: (A) GC-MS analysis of Vitex trifolia. In total, 89 components were recorded. The major components were eucalyptol, sabinen, and caryophyllene with respective peak areas of $16.35 \%, 9.44 \%$, and $8.91 \%$. The retention times were 10.03 , 19.39 , and 8.40 min. The percentage peak areas were calculated using Turbomass software, version 5.2.0. (B) GC-MS analysis for Vitex negundo. A total of 60 compounds were found. The primary component was found to be caryophyllene, with a peak area of $27.984 \%$ and a retention time of $16.48 \mathrm{~min}$. 
TABLE 1: The average mortality $( \pm \mathrm{SE})$, and $\mathrm{LC}_{50}$ and $\mathrm{LC}_{90}$ of Aedes aegypti and Culex quinquefasciatus larvae in the presence of Vitex trifolia and Vitex negundo leaves essential oil in vitro.

\begin{tabular}{|c|c|c|c|c|c|c|c|c|c|c|}
\hline \multirow{2}{*}{$\begin{array}{l}\text { Larval } \\
\text { stage }\end{array}$} & \multirow{2}{*}{$\begin{array}{l}\text { Larva } \\
\text { species }\end{array}$} & \multirow{2}{*}{$\begin{array}{c}\text { Essential } \\
\text { oil }\end{array}$} & \multicolumn{4}{|c|}{$\begin{array}{l}\text { Percentage mortality at each concentration } \\
\text { (PPM) }\end{array}$} & \multirow[b]{2}{*}{ Equation } & \multirow[b]{2}{*}{$\mathbf{R}^{2}$} & \multirow{2}{*}{$\begin{array}{l}\mathrm{LC}_{50} \\
(\mathrm{ppm})\end{array}$} & \multirow{2}{*}{$\begin{array}{l}\mathrm{LC}_{90} \\
(\mathrm{ppm})\end{array}$} \\
\hline & & & 50 & 75 & 100 & 125 & & & & \\
\hline $\begin{array}{c}3^{\text {rd }} \\
\text { instar }\end{array}$ & Aedes aegypti & Vitex trifolia & $29 \pm 2.3$ & $85 \pm 2.5$ & $96 \pm 2.0$ & $100 \pm 0.0$ & $\begin{array}{c}\mathrm{Y}= \\
76.22 \ln (X)- \\
259.1\end{array}$ & 0.873 & $57.77 \pm 0.4$ & $77.91 \pm 0.9$ \\
\hline $\begin{array}{l}3^{\text {rd }} \\
\text { instar }\end{array}$ & $\begin{array}{c}\text { Culex } \\
\text { quinquefasciatus }\end{array}$ & Vitex trifolia & $7.5 \pm 2.1$ & $78 \pm 1$ & $97 \pm 1.1$ & $100 \pm 0.0$ & $\begin{array}{c}Y= \\
66.54 \ln (X)- \\
215.0\end{array}$ & 0.937 & $55.17 \pm 3.14$ & $78.28 \pm 2.23$ \\
\hline
\end{tabular}

${ }^{*} p$-value $<0.05$, statistically significant difference in death rate for the concentrations tested using a chi square test.

From the results of the present study (Table 1), it is evident that the essential oil of $V$. trifolia and $V$. negundo demonstrated significant larvicidal activity against the third instar stages of Ae. aegypti. This study also showed a significant larvicidal action of Vitex trifolia against C. quinquefasciatus larvae. However, the activity of $V$. negundo on $C$. quinquefasciatus was not carried out because of the lack of sufficient Culex eggs during the study period. The percentage of larval mortality increased with increasing concentrations of the oil. Among the oils studied, $V$. negundo showed improved efficacy towards Ae. aegypti larvae; $\mathrm{The}_{\mathrm{LC}}$ and $\mathrm{LC}_{90}$ for this oil on these larvae were $50.86 \pm 0.9$ and $73.12 \pm 0.3 \mathrm{ppm}$ respectively. As per the classification established by Cheng et al. (2003), compounds with $\mathrm{LC}_{50}>100 \mathrm{mg} / \mathrm{mL}$ were considered not active, those with $\mathrm{LC}_{50}<100 \mathrm{mg} / \mathrm{mL}$ were active, and those with $\mathrm{LC}_{50}<50 \mathrm{mg} / \mathrm{mL}$ were highly active ${ }^{12}$. Therefore, based on the $\mathrm{LC}_{50}$ calculated in the present study, the essential oil of $V$. trifolia and $V$. negundo is considered to be active in controlling mosquito larvae.

The essential oil contains a blend of chemicals that can facilitate an increase or decrease in larvicidal activity, compared with the actions of their isolated constituents ${ }^{13}$. In most cases, the mosquito larvicidal activity of the blend is higher than those of its purified constituent compounds ${ }^{14,15}$. However, the individual compound or synergistic action of the studied oil is not known. A previous study has documented the presence of eucalyptol, a major compound in the essential oil of the leaves of $V$. trifolia. The GC-MS analysis of the purified oil carried out in the present study also confirmed these earlier results. The resultant spectra showed a major peak that corresponded to the eucalyptol compound, which occupies a total of $16.35 \%$ of all other compounds present in the oil (Figure 2A). Eucalyptol is reported to be present in the essential oil of other plants that exhibited larvicidal activity against mosquitoes ${ }^{16}$. Therefore, eucalyptol may contribute to the high larvicidal activity of this studied oil. However, the exact mechanisms of the oil's larvicidal property needs further study into a more extensive range of mosquito species.

In conclusion, we found from this study that the essential oil of $V$. trifolia possesses potent larvicidal activity. The oil showed $100 \%$ mortality against third instar stages of Ae. aegypti and
C. quinquefasicatus larvae at a lower concentration of $125 \mathrm{ppm}$. The oil could be used separately or in combination with existing larvicides, creating new effective eco-friendly and affordable approaches for effective control of disease-transmitting vector mosquitoes.

\section{ACKNOWLEDGEMENTS}

We sincerely thank the honorable Vice-chancellor and Dean from the Sponsored Research department of SASTRA Deemed University, Thanjavur, India, for facilities and encouragement. We are grateful to Dr. P. Brindha, Associate Dean, CARISM, SASTRA Deemed University for granting permission to collect samples and for the GC-MS facilities.

\section{Conflict of interest}

The authors declare that they have no conflict of interests.

\section{REFERENCES}

1. Ghosh A, Chowdhury N, Chandra G. Plant extracts as potential mosquito larvicides. The Indian journal of medical research. 2012;135(5):581.

2. Wahyuni D. Larvicidal Activity of Essential Oils of Piper betle from the Indonesian Plants against Aedes aegypti L. 2012.

3. Mathew K. The Flora of the Tamil Nadu Carnatic, The Rapinet herbarium, St. Joseph's college, Tiruchirapalli, India. 1983.

4. Organization WH. Guidelines for laboratory and field testing of mosquito larvicides. Geneva: World Health Organization, 2005.

5. Vincent K. Probit analysis. San Francisco: San Francisco State University. 2008.

6. Warrier PK, Nambiar V. Indian medicinal plants: a compendium of 500 species: Orient Blackswan; 1993.

7. Kannathasan K, Senthilkumar A, Chandrasekaran M, Venkatesalu V. Differential larvicidal efficacy of four species of Vitex against Culex quinquefasciatus larvae. Parasitology research. 2007;101(6):1721-3.

8. Kannathasan K, Senthilkumar A, Venkatesalu V, Chandrasekaran M. Larvicidal activity of fatty acid methyl esters of Vitex species against Culex quinquefasciatus. Parasitology research. 2008;103(4):999-1001. 
9. Kannathasan K, Senthilkumar A, Venkatesalu V. Mosquito larvicidal activity of methyl-p-hydroxybenzoate isolated from the leaves of Vitex trifolia Linn. Acta Tropica. 2011;120(1-2):115-8.

10. Tawatsin A, Asavadachanukorn P, Thavara U, Wongsinkongman P, Bansidhi $\mathrm{J}$, Boonruad T, et al. Repellency of essential oils extracted from plants in Thailand against four mosquito vectors (Diptera: Culicidae) and oviposition deterrent effects against Aedes aegypti (Diptera: Culicidae). Southeast Asian journal of tropical medicine and public health. 2006;37(5):915.

11. Killeen GF, Fillinger U, Kiche I, Gouagna LC, Knols BG. Eradication of Anopheles gambiae from Brazil: lessons for malaria control in Africa? The Lancet infectious diseases. 2002;2(10):618-27.

12. Cheng S-S, Chang H-T, Chang S-T, Tsai K-H, Chen W-J. Bioactivity of selected plant essential oils against the yellow fever mosquito Aedes aegypti larvae. Bioresource Technology. 2003;89(1):99-102.

13. Waliwitiya R, Kennedy CJ, Lowenberger CA. Larvicidal and oviposition-altering activity of monoterpenoids, trans-anithole and rosemary oil to the yellow fever mosquito Aedes aegypti (Diptera: Culicidae). Pest Management Science: formerly Pesticide Science. 2009;65(3):241-8.

14. Lahlou M. Methods to study the phytochemistry and bioactivity of essential oils. Phytotherapy Research: An International Journal Devoted to Pharmacological and Toxicological Evaluation of Natural Product Derivatives. 2004;18(6):435-48.

15. Shaalan EA-S, Canyon DV, Younes MWF, Abdel-Wahab H, Mansour A-H. Effects of sub lethal concentrations of synthetic insecticides and Callitris glauscophylla extracts on the development of Aedes aegypti. Journal of Vector Ecology. 2005;30:295-8.

16. Zhu L, Tian Y. Chemical composition and larvicidal activity of essential oil of Artemisia gilvescens against Anopheles anthropophagus. Parasitology research. 2013;112(3):1137-42. 